\title{
Effet de l'apport de lipides sur les flux d'azote duodénal chez la vache
}

\author{
M Doreau ${ }^{1}, \mathrm{~F}$ Legay $^{2}$, A Ferlay $^{1}$, D Bauchart ${ }^{2}$ \\ 1 Laboratoire de la lactation et de l'élevage des ruminants; \\ 2 Unité de recherches sur le métabolisme énergétique et lipidique, \\ INRA Theix, F63122 Saint-Genès-Champanelle, France
}

\begin{abstract}
Summary - Influence of lipid supply in the diet on the nitrogen flow in the duodenum of cows. Lipid supplementation of dairy rations did not significantly modify duodenal flows of dietary nitrogen, liquid-associated and solid-adherent bacterial nitrogen when organic matter ruminal digestion was not modified.
\end{abstract}

L'addition de lipides dans la ration du ruminant a souvent pour effet de diminuer la quantité de matière organique digérée dans le rumen; ceci ne limiterait pas la synthèse de protéines microbiennes, peut-être grâce à une amélioration de l'efficacité de cette synthèse dans le rumen. Les résultats de la bibliographie sont cependant peu cohérents, probablement en raison de la difficulté de la détermination du flux duodénal d'azote bactérien.

Matériel et méthodes - Deux essais ont été réalisés sur vaches Holstein en production, munies de canules du rumen et du duodénum. Quatre vaches ont reçu 4 régimes $\mathrm{T} 1, \mathrm{C}_{1}, \mathrm{C}_{2}$ et $\mathrm{S}$, selon un carré latin $4 \times 4$ (essai 1). Trois vaches ont reçu 3 régimes T2, GC et GE, selon un carré latin $3 \times 3$ (essai 2). Les rations témoins $\mathrm{T} 1$ et $\mathrm{T} 2$ comprenaient environ $60 \%$ de foin et $40 \%$ d'aliment concentré. Les rations enrichies en matières grasses avaient la même composition de base, mais étaient additionnées de matières grasses : huile de colza à 2 niveaux d'incorporation ( $\mathrm{C} 1$ et $\mathrm{C} 2$ ); suif (S); graine de colza crue (GC) ou extrudée (GE). Les rations, distribuées en quantités limitées cou- vrant les besoins des animaux, étaient calculées de manière que, pour un même essai, les mêmes quantités de foin et d'aliment concentré soient apportées par l'ensemble des régimes. Les teneurs en acides gras des rations étaient de 2,0 (T1), 5,5 (C1), 9,4 (C2), 10,1 (S), 4,2 (T2), 8,9 (GC) et 8,9 (GE) $\%$ de la matière sèche (MS). L'apport total de calcium atait de $1 \%$ environ de la ration totale.

Le flux duodénal de MS a été déterminé grâce à des infusions ruminales continues et simultanées de 3 marqueurs : chlorure d'ytterbium, oxyde de chrome et polyéthylène glycol ( $P E G$ ), utilisés en simple marquage. La vitesse de renouvellement du liquide du rumen a été mesurée par la cinétique de décroissance du PEG (essai 1) ou du cobalt-EDTA (essai 2) dans le rumen. Le flux duodénal bactérie a été obtenu selon Legay-Carmier et al (1988) à partir de la vitesse de sortie de l'eau libre du rumen et du compartiment de bactéries libres ruminales pour les bactéries de la phase liquide, et à partir des concentrations en ARN des bactéries et du contenu duodénal pour les bactéries fixées aux particules du rumen. Les matières azotées totales ont été analysées sur des échantillons représentatifs des bactéries libres et fixées et de contenu duodénal 
Tableau I. Ingestion et digestion ruminale de la matière organique (MO), et bilan de l'azote ingéré et duodénal.

\begin{tabular}{|c|c|c|c|c|c|c|c|}
\hline \multirow{2}{*}{ Régime } & \multicolumn{4}{|c|}{ Essai 1} & \multicolumn{3}{|c|}{ Essai 2} \\
\hline & $T 1$ & $C 1$ & $\mathrm{C} 2$ & $S$ & $T 2$ & $G C$ & $G E$ \\
\hline $\begin{array}{l}\text { MO ingérée }(\mathrm{kg} / \mathrm{j}) \\
\text { MODR (kg/j) } \\
N \text { ingéré }(\mathrm{g} / \mathrm{j}) \\
\text { Flux de } N \text { NA duodénal }(g / j)\end{array}$ & $\begin{array}{r}14,0 \\
7,3 \\
413\end{array}$ & $\begin{array}{r}14,9 \\
7,8 \\
413\end{array}$ & $\begin{array}{r}15,0 \\
7,7 \\
415\end{array}$ & $\begin{array}{r}14,4 \\
7,9 \\
395\end{array}$ & $\begin{array}{r}13,5 \\
9,1 \\
602\end{array}$ & $\begin{array}{r}14,5 \\
8,7 \\
596\end{array}$ & $\begin{array}{r}14,5 \\
9,1 \\
605\end{array}$ \\
\hline $\begin{array}{l}\text { total } \\
\text { de bactéries libres } \\
\text { de bactéries fixées }\end{array}$ & $\begin{array}{r}402 \\
88 \\
154\end{array}$ & $\begin{array}{l}396 \\
106 \\
172\end{array}$ & $\begin{array}{r}403 \\
98 \\
160\end{array}$ & $\begin{array}{l}373 \\
100 \\
138\end{array}$ & $\begin{array}{l}454 \\
137 \\
191\end{array}$ & $\begin{array}{l}462 \\
129 \\
203\end{array}$ & $\begin{array}{l}453 \\
139 \\
154\end{array}$ \\
\hline
\end{tabular}

par la méthode de Kjeldahl. Les concentrations en ytterbium, cobalt et chrome ont été déterminées par spectrophotométrie d'absorption atomique, la teneur en PEG par turbidimétrie, la teneur en ARN par analyse colorimétrique des bases puriques.

Résultats et discussion - Le flux total d'azote non ammoniacal au niveau duodénal (NNA) n'a pas été modifié par l'apport de matières grasses, pour un même essai. De même, la supplémentation lipidique n'a pas significativement modifié le flux d'azote "alimentaire" (calculé par différence entre flux total et flux bactérien, et qui correspond en réalité à la somme des flux alimentaire et endogène). Le flux d'azote bactérien a représenté de 69 à $82 \%$ du flux total d'azote; aucun effet significatif de matières grasses n'a été mis en évidence sur les flux de bactéries libres et fixées. L'efficacité de la synthèse microbienne n'a pas été accrue par la supplémentation lipidique, contrairement aux résultats de Sutton et al (1983). La raison est peut-être que la biomasse de protozoaires a été peu modifiée (Bauchart et al, 1988) et la quantité de matière organique apparemment digestible dans le rumen (MODR) non réduite, et donc que, dans ces essais, les lipides n'ont pas eu d'action sur la microflore ruminale. Le rapport $\mathrm{N}$ bactérien duodénal / MODR a été compris entre 30 et $36 \mathrm{~g} / \mathrm{kg}$ dans l'essai 1,32 et $38 \mathrm{~g} / \mathrm{kg}$ dans l'essai 2.

En conclusion, dans des essais où la digestibilité ruminale de la matière organique n'a pas été affectée, malgré un apport important de lipides, les flux d'azote total et microbien ont été peu modifiés.

Bauchart D, Legay-Carmier F, Jouany JP, Michalet-Doreau B, Doreau M (1988) Reprod Nutr Dév 28 (suppl 1), 85-86

Legay-Carmier F, Bauchart D, Doreau M (1988) Reprod Nutr Dév 28 (suppl 1), 117-118

Sutton JD, Knight R, McAllan AB, Smith RH (1983) $\mathrm{Br}$ J Nutr 49, 419-432 